\title{
IMPROVED STRENGTH AND RESISTANCE
}

GC Europe, a leader in dental materials technology, is pleased to announce the launch of two superior flowable materials: G-ænial Universal Flo and G-ænial Flo, to give patients the best aesthetic, functional and durable restorations.

Both flowable materials benefit from a composition that features a unique filler technology. Unlike other flowable composites the GC materials have a higher filler load and a homogeneous dispersion of fillers. The resulting improved strength and wear resistance are two key features of these flowable materials, opening up the potential for a broader use than standard flowables.

G-ænial Universal Flo is radiopaque and features a high viscosity, making it ideal for placement in class I-V restorations. Essentially, it looks like a flowable but behaves like a restorative.
Its indications are for direct restorations, minimum intervention cavities and fissure sealing. With a physical performance of a regular composite, this material is highly thixotropic and stays neatly in place holding its shape for ease of use. You will find it to be virtually self-polishing with superb invisible aesthetics.

These restoratives are dispensed through an ergonomically designed syringe that enables smooth delivery of the material, with a tapered tip that means no paste can stick to it. The syringe size provides a comfortable hold and the arched shape of the flange fits the hand ergonomically. The design also prevents material waste because minimal residual paste remains in the syringe after use.

\section{Reader response number $\mathbf{5 7}$}

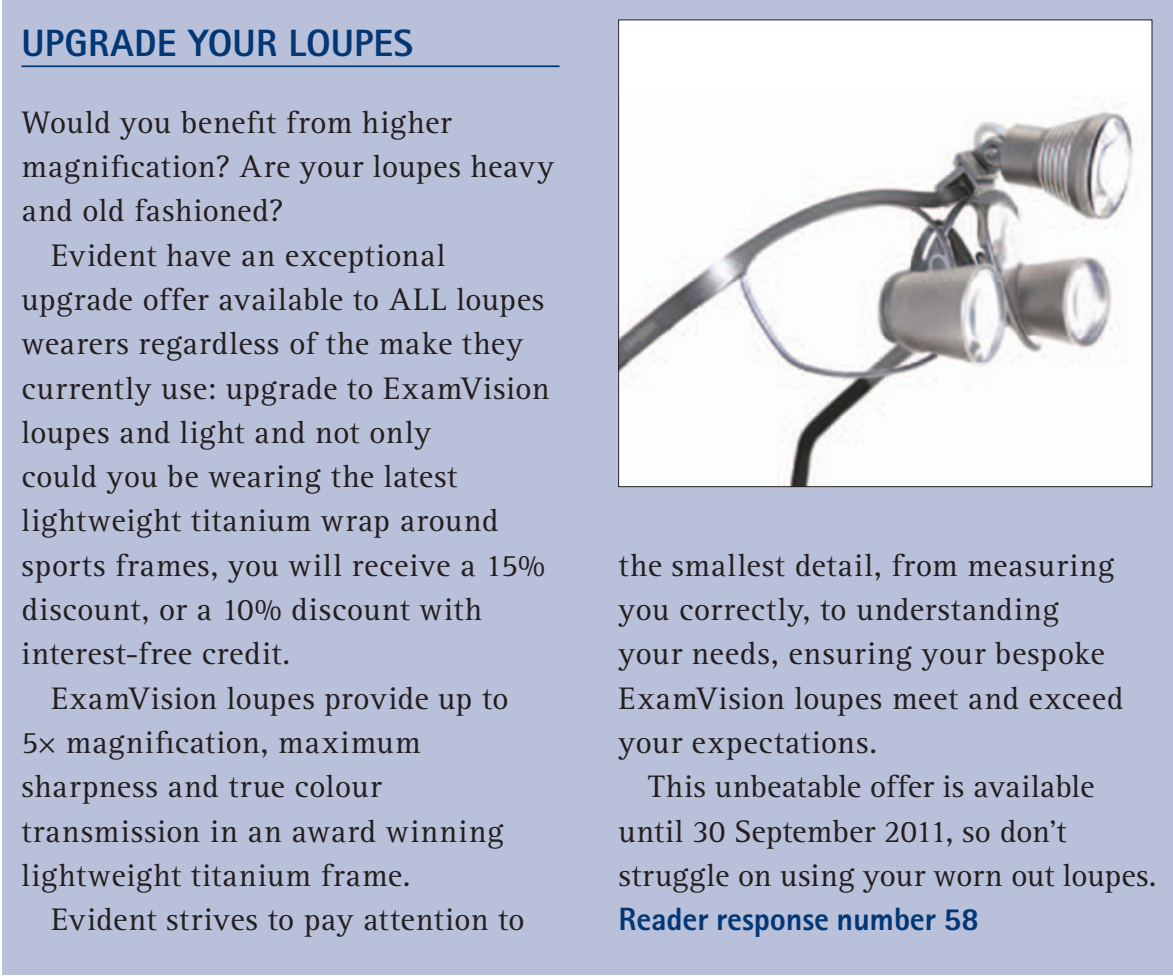

\section{PROVIDE A BRILLIANT SMILE}

As every cosmetic dentist knows, the fit of a whitening tray is essential in creating the very best aesthetic results for your patient. S4S can help your patients achieve the smile of their dreams with its innovative range of whitening devices, such as its bleaching retainer with reservoirs for placing whitening gels.

S4S offer a comprehensive range of custom-made trays that will not only fit perfectly, but will also suit virtually every type of gel and whitening system, providing your patients with a brilliant smile they can be proud of.

Reader response number 59 